DOI: $10.17516 / 1998-2836-0183$

УДК 547.1’13:546.725:542.06

\title{
Pd/Cu-Catalyzed Coupling of Cyclopentadienyliron Dicarbonyl Iodide and Methyl Propiolate in Synthesis of $\mathrm{Cp}(\mathrm{CO})_{2} \mathrm{Fe}-\mathrm{C} \equiv \mathrm{C}-\mathrm{COOMe}$
}

\author{
Victor V. Verpekin* and Alexander A. Kondrasenko \\ Institute of Chemistry and Chemical Technology SB RAS \\ FRC "Krasnoyarsk Science Center SB RAS" \\ Krasnoyarsk, Russian Federation
}

Received 29.05.2020, received in revised form 01.06.2020, accepted 07.06.2020

Abstract. The conditions of successful iron-carbon coupling in the reaction between cyclopentadienyliron dicarbonyl iodide and methyl propiolate upon the $\mathrm{Pd} / \mathrm{Cu}$ catalysis were determined. A new high-yield synthetic approach to the methoxycarbonylethynyl iron complex $\mathrm{Cp}(\mathrm{CO})_{2} \mathrm{Fe}-\mathrm{C} \equiv \mathrm{C}-\mathrm{COOMe}$ were developed.

Keywords: iron, palladium, copper, iron-carbon coupling, acetylide complexes.

Citation: Verpekin V.V., Kondrasenko A.A. Pd/Cu-catalyzed coupling of cyclopentadienyliron dicarbonyl iodide and methyl propiolate in synthesis of $\mathrm{Cp}(\mathrm{CO})_{2} \mathrm{Fe}-\mathrm{C} \equiv \mathrm{C}-\mathrm{COOMe}$, J. Sib. Fed. Univ. Chem., 2020, 13(2), 297-303. DOI: 10.17516/1998-28360183

(C) Siberian Federal University. All rights reserved

This work is licensed under a Creative Commons Attribution-NonCommercial 4.0 International License (CC BY-NC 4.0).

* Corresponding author E-mail address:vvv@icct.ru,vvv@sany-ok.ru 


\title{
Синтез $\mathrm{Cp}(\mathrm{CO})_{2} \mathrm{Fe}-\mathrm{C} \equiv \mathrm{C}-\mathrm{COOMe}$ \\ через $\mathrm{Pd} / \mathrm{Cu}$-катализируемое сочетание \\ циклопентадиенилдикарбонилиодида железа \\ и метилпропиолата
}

\author{
В.В. Верпекин, А.А. Кондрасенко \\ Институт химии и химической технологии СО РАН \\ ФИЦ «Красноярский научный центр СО РАН» \\ Российская Федереиия, Красноярск
}

\begin{abstract}
Аннотация. Найдены оптимальные условия для проведения реакции сочетания железо-углерод между циклопентадиенилдикарбонилиодидом железа и метилпропиолатом в условиях $\mathrm{Pd} / \mathrm{Cu}$ катализа. Предложена новая методика синтеза этинилметоксикарбонильного комплекса железа $\mathrm{Cp}(\mathrm{CO})_{2} \mathrm{Fe}-\mathrm{C} \equiv \mathrm{C}-\mathrm{COOMe}$, позволяющая получать его с количественными выходами.
\end{abstract}

Ключевые слова: железо, палладий, медь, сочетание железо-углерод, ацетиленидные комплексы.

Цитирование: Верпекин, В.В. Синтез $\mathrm{Cp}(\mathrm{CO})_{2} \mathrm{Fe}-\mathrm{C} \equiv \mathrm{C}-\mathrm{COOMe}$ через $\mathrm{Pd} / \mathrm{Cu}$-катализируемое сочетание циклопентадиенилдикарбонилиодида железа и метилпропиолата / В.В. Верпекин, А.А. Кондрасенко // Журн. Сиб. федер. ун-та. Химия, 2020. 13(2). С. 297-303. DOI: 10.17516/1998-2836-0183

\section{Введение}

Переходные металлы, содержащие б-алкинильные и $\sigma$-полииновые лиганды, обычно используют как модельные системы для изучения люминесценции, нелинейно-оптических свойств и процессов переноса электронов на молекулярном уровне. В последнее время их также начали рассматривать как прекурсоры для создания функциональных материалов [1-4] и катализаторов процессов восстановления протонов до водорода [5, 6]. В связи с этим разработка методов, позволяющих селективно получать $\sigma$-алкинильные комплексы с максимальными выходами, является актуальной [7].

Наиболее перспективны подходы, основанные на использовании катализируемых реакций сочетания металл-алкинил. Для проведения таких реакций в качестве катализаторов могут использоваться соединения меди [8], золота [9], палладия [10], а в качестве источников $\sigma$-алкинильных лигандов - терминальные алкины, триалкилстанилацетилены, триметилсилилацетилены. Эти методы позволяют проводить реакции сочетания металл-углерод с высокими выходами в стандартных условиях, с использованием стабильных исходных веществ. Так, группой Коитиро Ошимы было предложено использовать металлоорганический аналог $\mathrm{Pd} / \mathrm{Cu}-$ катализируемых реакций сочетания Соногаширы, позволяющий с высокими выходами получать арилэтинильные комплексы железа [11]. Однако протокол Ошимы оказался неприменим в некоторых случаях, например, реакция орто-пиридилэтинила с $\mathrm{Cp}(\mathrm{CO})_{2} \mathrm{FeI}$ привела не к ожидаемому комплексу $\mathrm{Cp}(\mathrm{CO})_{2} \mathrm{Fe}-\mathrm{C} \equiv \mathrm{C}-\left(2-\mathrm{C}_{5} \mathrm{H}_{4} \mathrm{~N}\right)$, а к необычному биядерному $\mathrm{FePd}$ 
комплексу с мостиковым 2-пиридилвинилиденовым лигандом $\left[\mathrm{Cp}(\mathrm{CO}) \mathrm{Fe}\left\{\mu_{2}-\eta^{1}\left(\mathrm{C}_{\alpha}\right): \eta^{1}\left(\mathrm{C}_{\alpha}\right)\right.\right.$ $\left.\left.\kappa^{1}(\mathrm{~N})-\mathrm{C}_{\alpha}=\mathrm{C}_{\beta}(\mathrm{H})\left(2-\mathrm{C}_{5} \mathrm{H}_{4} \mathrm{~N}\right)\right\}(\mu-\mathrm{CO}) \mathrm{PdI}\right][12]$. В связи с этим нами был разработан эффективный подход к синтезу пиридилэтинильных $\mathrm{Cp}(\mathrm{CO})_{2} \mathrm{Fe}-\mathrm{C} \equiv \mathrm{C}-\left(\mathrm{n}-\mathrm{C}_{5} \mathrm{H}_{4} \mathrm{~N}\right)(\mathrm{n}=$ opmo-, мета-, napa-) и 4-(2,1,3-бензотиадиазолил)-этинильного $\mathrm{Cp}(\mathrm{CO})_{2} \mathrm{Fe}-\mathrm{C} \equiv \mathrm{C}-\left(4-\mathrm{C}_{6} \mathrm{H}_{3} \mathrm{~N}_{2} \mathrm{~S}\right)$ комплексов [13]. Чтобы оценить возможности использования разработанного нами подхода для синтеза других $\sigma$-алкинилов переходных металлов, в этой работе изучены закономерности протекания реакций сочетания $\mathrm{Cp}(\mathrm{CO})_{2} \mathrm{FeI}$ и $\mathrm{H}-\mathrm{C} \equiv \mathrm{C}-\mathrm{COOMе} \mathrm{в} \mathrm{условиях} \mathrm{Pd} / \mathrm{Cu}$ и $\mathrm{Pd}$ катализа, которые привели к образованию известного этинилметоксикарбонильного комплекса железа $\mathrm{Cp}(\mathrm{CO})_{2} \mathrm{Fe}-\mathrm{C} \equiv \mathrm{C}$ COOMe (1).

\section{Экспериментальная часть}

Все операции по синтезу и выделению комплекса проводили в атмосфере аргона. Растворители (этилацетат, петролейный эфир, хлористый метилен) предварительно очищали от примесей, следов воды и кислорода путем перегонки над соответствующими осушителями и хранили в атмосфере аргона. Тетрагидрофуран (ТГФ) для проведения реакций осушали над дифенилкетилом натрия и перегоняли в атмосфере аргона непосредственно перед применением. Мониторинг реакций проводили с использованием тонкослойной хроматографии на пластинках со слоем силикагеля (Alu foils, Sigma-Aldrich) и ИК-спектроскопии. Для хроматографического разделения реакционных смесей применяли нейтральный силикагель (Silica 60, 0,2-0,5 mm) фирмы Macherey-Nagel. В экспериментах использовали 1,8-диазабицикло[5.4.0] ундец-7-ен (ДБУ, Aldrich), диизопропиламин, метилпропиолат (Acros Organics) и йодид меди (ООО «Вектон-М»). Бис(ацетонитрил)дихлор палладий (II) $\mathrm{PdCl}_{2}\left(\mathrm{NCMe}_{2}\right.$, циклопентадиенилдикарбонилиодид железа $\mathrm{Cp}(\mathrm{CO})_{2} \mathrm{FeI}$ были синтезировали по ранее опубликованным методикам $[14,15]$.

Физико-химические характеристики получены с помощью оборудования Красноярского регионального центра коллективного пользования ФИЦ КНЦ СО РАН. Спектры ИК записаны на спектрометре Shimadzu IRTracer-100 (Japan). Спектры ЯМР ${ }^{1} \mathrm{H}$ и ${ }^{13} \mathrm{C}$ были получены с использованием Фурье-спектрометра ЯMP AVANCEIII 600 (Bruker, Германия). Химические сдвиги в спектрах определены относительно остаточных протонов дейтерированных растворителей и приведены в миллионных долях (м.д.). Константы спин-спиновых взаимодействий приведены в герцах (Гц).

В экспериментальной части описана только методика, позволяющая получить $\mathrm{Cp}(\mathrm{CO})_{2} \mathrm{Fe}-$ $\mathrm{C} \equiv \mathrm{C}-\mathrm{COOMе} \mathrm{с} \mathrm{максимальным} \mathrm{выходом.} \mathrm{Синтез} \mathrm{и} \mathrm{выделение} \mathrm{комплекса} 1$ по остальным методикам проводили аналогичным образом.

К раствору циклопентадиенилдикарбонилиодида железа (315 мг, 1,036 ммоль) и метилпропиолата (0,155 мл, 158 мг, 1,534 ммоль) в 10 мл свежеперегнанного тетрагидрофурана при интенсивном перемешивании добавляли 0,23 мл (234 мг, 1,539 ммоль) ДБУ, затем навески катализаторов $\mathrm{PdCl}_{2}(\mathrm{NCMe})_{2}$ (3 мг, 0,012 ммоль, 1 моль \%) и $\mathrm{CuI}$ (10 мг, 0,052 ммоль, 5 моль \%). Реакционную смесь перемешивали в течение 30 мин при $60^{\circ} \mathrm{C}$, затем упаривали в вакууме, перерастворяли в дихлорметане и профильтровывали через силикагель ( 0,5 см), используя этилацетат как элюент. Фильтрат сконцентрировали и нанесли на хроматографическую колонку с нейтральным $\mathrm{SiO}_{2}(9 \times 2$ см). Смесью петролейный эфир-дихлорметан (9:1) и (4:1) элюирова- 
ли первую фракцию, содержащую (9 мг, 0,003 ммоль) $\mathrm{Cp}(\mathrm{CO})_{2} \mathrm{FeI}$. Вторую красную фракцию, содержащую (6 мг, 0,017 ммоль, 3 \%) $\left[\mathrm{Cp}(\mathrm{CO})_{2} \mathrm{Fe}\right]_{2}$, элюировали смесью петролейный эфир-дихлорметан (7:3). Третью, желто-коричневую фракцию элюировали смесью петролейный эфирэтилацетат (3:7), из которой после удаления растворителя было получено 248 мг (0,954 ммоль, 92 \%) желто-коричневого порошка комплекса $\mathrm{Cp}(\mathrm{CO})_{2} \mathrm{Fe}-\mathrm{C} \equiv \mathrm{C}-\mathrm{COOMe}(\mathbf{1})$.

${ }^{1} \mathrm{H}$ ЯMP( $\left(\mathrm{CDCl}_{3}, 25{ }^{\circ} \mathrm{C}\right) \delta$, м.д.: 3,68 (c, 3H, $\left.-\mathrm{CH}_{3}\right) ; 5,09\left(\mathrm{c}, 5 \mathrm{H}, \mathrm{C}_{5} \boldsymbol{H}_{5}\right) \cdot{ }^{13} \mathrm{C} \mathrm{NMR}\left(\mathrm{CDCl}_{3}, 25{ }^{\circ} \mathrm{C}\right) \delta$, м.д.: 51,9 (c, $\left.-\boldsymbol{C} \mathrm{H}_{3}\right) ; 85,6$ (c, $\left.\boldsymbol{C}_{5} \mathrm{H}_{5}\right) ; 107,9$ (c, $\equiv \boldsymbol{C}^{2}$ ); 108,2 (c, $\left.-\boldsymbol{C}^{\mathrm{l}} \equiv\right) ; 152,5$ (c, $\left.-\boldsymbol{C}=\mathrm{O}\right) ; 210,8$ (c, 2Fe- $\boldsymbol{C O}$ ). IR $\left(\mathrm{CH}_{2} \mathrm{Cl}_{2} v / \mathrm{cm}^{-1}\right): 2113 \mathrm{c}\left(v_{\mathrm{C}=\mathrm{C}}\right), 2047 \mathrm{oc}, 2005 \mathrm{oc}\left(v_{\mathrm{CO}}\right), 1684 \mathrm{c}\left(v_{\mathrm{C}=0}\right)$. Элементный анализ. Найдено: C, 50,67 \%; Н, 3,09 \%. Рассчитано для $\mathrm{C}_{11} \mathrm{H}_{8} \mathrm{FeO}_{4}(260)$ : C, 50,81 \%; Н, 3,10 \%.

\section{Результаты и обсуждение}

$\mathrm{B}$ нашей предыдущей работе [13] на примере изучения закономерностей протекания $\mathrm{Pd} / \mathrm{Cu}-$ и Pd-катализируемых реакций сочетания циклопентадиенилдикарбонилиодида железа с терминальными пиридилацетиленами было показано, что эти реакции являются гетероаналогами классических реакций сочетания углерод-углерод [16] и, вероятнее всего, протекают как последовательность стадий окислительного присоединения $\mathrm{Cp}(\mathrm{CO})_{2} \mathrm{FeI}$ к каталитически активному комплексу палладия по связи Fe-I, переноса алкинильного фрагмента к атому палладия (трансметаллирование) и завершающего Fе-С восстановительного элиминирования (рис. 1).

Было установлено, что для получения пиридилэтинильных комплексов $\mathrm{Cp}(\mathrm{CO})_{2} \mathrm{Fe}-\mathrm{C} \equiv \mathrm{C}$ $\left(\mathrm{n}-\mathrm{C}_{5} \mathrm{H}_{4} \mathrm{~N}\right)(\mathrm{n}=$ орто (2), мета (3), пара (4)) с максимальными выходами могут быть использованы два варианта условий проведения этих реакций сочетания: 1) при комнатной темпе-

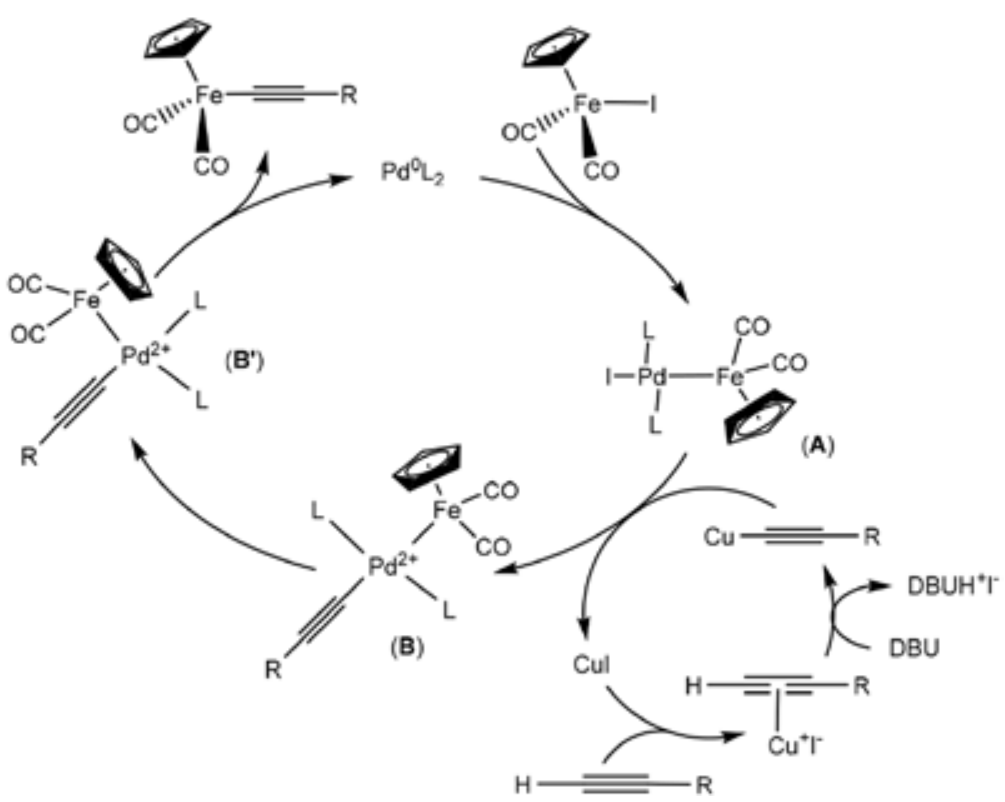

Рис. 1. Предполагаемый механизм Pd/Cu-катализируемой реакции сочетания циклопентадиенилдикарбонилиодида железа с терминальными алкинами

Fig. 1. Proposed mechanism of $\mathrm{Pd} / \mathrm{Cu}$-catalyzed coupling reactions of cyclopentadienyliron dicarbonyl iodide and terminal alkynes 
ратуре в присутствии 2 моль \% $\mathrm{PdCl}_{2}(\mathrm{NCMe})_{2}, 20$ моль \% $\mathrm{CuI}$ в течение 20 мин (условия 1); 2) с использованием уменьшенных навесок катализаторов (1 моль \% $\mathrm{PdCl}_{2}(\mathrm{NCMe})_{2}$ и 5 моль \% $\mathrm{CuI})$ и повышенной температуры реакционной смеси до $60{ }^{\circ} \mathrm{C}$ в течение 30 мин (условия 2). В качестве растворителя во всех реакциях использовался тетрагидрофуран, а в качестве основания - 1,8-диазобицикло[5.4.0]ундец-7-ен (ДБУ). При этом основным фактором, обеспечивающим протекание реакций сочетания $\mathrm{Cp}(\mathrm{CO})_{2} \mathrm{FeI}$ с орто-, мета- и пара-пиридилацетиленами, оказалось применение ДБУ, так как использование в этих реакциях более слабых оснований, таких как триэтиламин или триизопропиламин, не приводило к образованию целевых пиридилэтинильных комплексов железа 2-4.

Для изучения возможности синтеза $\mathrm{Cp}(\mathrm{CO})_{2} \mathrm{Fe}-\mathrm{C} \equiv \mathrm{C}-\mathrm{COOMe}(\mathbf{1})$ по реакции катализируемого сочетания $\mathrm{Cp}(\mathrm{CO})_{2} \mathrm{FeI}$ и $\mathrm{H}-\mathrm{C} \equiv \mathrm{C}-\mathrm{COOMe}$ были использованы: 1) предложенные нами ранее условия $\mathrm{Pd} / \mathrm{Cu}$ катализа; 2) протокол Ошимы (с использованием в качестве палладиевого катализатора $\mathrm{PdCl}_{2}\left(\mathrm{NCMe}_{2}\right.$ вместо $\mathrm{PdCl}_{2}\left(\mathrm{PPh}_{3}\right)_{2}$ [11]; 3) Рd-катализируемая реакция без использования сокатализатора СuI. Условия реакций и полученные результаты по синтезу $\mathrm{Cp}(\mathrm{CO})_{2} \mathrm{Fe}-$ $\mathrm{C} \equiv \mathrm{C}-\mathrm{COOMе} \mathrm{(1)} \mathrm{приведены} \mathrm{в} \mathrm{табл.} 1$.

Как видно из полученных результатов, использование предложенных нами условий для проведения $\mathrm{Pd} / \mathrm{Cu}$-катализируемой реакции сочетания $\mathrm{Cp}(\mathrm{CO})_{2} \mathrm{FeI}$ и $\mathrm{H}-\mathrm{C} \equiv \mathrm{C}-\mathrm{COOMe}$ позволило получить комплекс $\mathbf{1}$ с количественными выходами, однако при использовании условий 1 выход целевого продукта составил 84 \%, а при использовании условий 2 - 92 \%; конверсия исходного циклопентадиенилдикарбонилиодида железа также отличалась примерно на 10 \% в обеих реакциях. В случае синтеза пиридиэтинильных комплексов 2-4 оба варианта проведения реакций давали примерно одинаковые результаты. Также в отличие от ранее изученных реакций пиридилэтинилирования $\mathrm{Cp}(\mathrm{CO})_{2} \mathrm{FeI}$ оказалось, что сочетание $\mathrm{Cp}(\mathrm{CO})_{2} \mathrm{FeI}$ и $\mathrm{H}-\mathrm{C} \equiv \mathrm{C}-\mathrm{COOMе} \mathrm{протекает} \mathrm{и} \mathrm{при} \mathrm{применении} \mathrm{условий} \mathrm{Ошимы,} \mathrm{хотя} \mathrm{выход} \mathrm{комплекса} 1$ в этом случае составил только 51 \%. В случае отсутствии йодида меди выход целевого комплекса 1 уменьшается до 53 \% (табл. 1, реакция 4), подобное уменьшение степени конверсии и

Таблица 1. Условия и результаты реакций синтеза $\mathrm{Cp}(\mathrm{CO})_{2} \mathrm{Fe}-\mathrm{C} \equiv \mathrm{C}-\mathrm{COOMe}(\mathbf{1})^{a}$

Table 1. Conditions and yield of the $\mathrm{Cp}(\mathrm{CO})_{2} \mathrm{Fe}-\mathrm{C} \equiv \mathrm{C}-\mathrm{COOMe}(\mathbf{1})$ synthesis $^{a}$

\begin{tabular}{|c|c|c|c|c|c|c|c|}
\hline \multirow{2}{*}{ \# } & \multirow{2}{*}{$\begin{array}{c}\mathrm{PdCl}_{2}(\mathrm{NCMe})_{2}, \\
\text { моль } \%\end{array}$} & \multirow{2}{*}{$\begin{array}{c}\mathrm{CuI}, \\
\text { моль \% }\end{array}$} & \multirow{2}{*}{ Основание } & \multirow{2}{*}{ Условия } & \multirow{2}{*}{$\begin{array}{c}\text { Конверсия }^{b}, \\
\%\end{array}$} & \multicolumn{2}{|c|}{ Выходы, \% } \\
\hline & & & & & & 1 & {$\left[\mathrm{Cp}(\mathrm{CO})_{2} \mathrm{Fe}\right]_{2}$} \\
\hline 1 & 2 моль \% & 20 & DBU (1,5 экв) & ТГФ, $24{ }^{\circ} \mathrm{C}, 20$ мин & 86 & 84 & - \\
\hline 2 & 1 моль \% & 5 & DBU (1,5 экв) & ТГФ, $60{ }^{\circ} \mathrm{C}, 30$ мин & 97 & 92 & 3 \\
\hline 3 & 2,5 моль \% & 5 & ${ }^{\mathrm{i}} \mathrm{Pr}_{2} \mathrm{NH}$ (4 мл); & ТГФ (8 мл), $24^{\circ} \mathrm{C}, 30$ мин & 60 & 51 & - \\
\hline 4 & 10 моль \% & - & DBU (1,5 экв) & ТГФ, $24{ }^{\circ} \mathrm{C}, 30$ мин & 57 & 53 & - \\
\hline
\end{tabular}

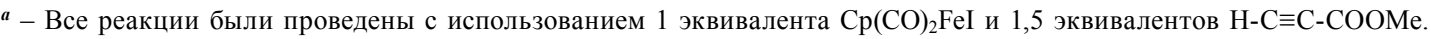

$b$ - Конверсия рассчитана на основе количества исходного $\mathrm{Cp}(\mathrm{CO})_{2} \mathrm{FeI}$, выделенного после хроматографии реакционной смеси. 
выходов пиридилэтинильных комплексов железа 2-4 в Рd-катализируемых условиях наблюдалось нами ранее. Стоит отметить, что ранее при проведении Pd-катализируемых реакций сочетания $\mathrm{Cp}(\mathrm{CO})_{2} \mathrm{FeI}$ и орто-пиридилэтинила, а также в условиях Ошимы (без образования $\left.\mathrm{Cp}(\mathrm{CO})_{2} \mathrm{Fe}-\mathrm{C} \equiv \mathrm{C}-\left(2-\mathrm{C}_{5} \mathrm{H}_{4} \mathrm{~N}\right)(2)\right)$ происходило протекание побочных реакций, приводящих к выделению FePd комплекса [Cp(CO)Fe $\left.\left\{\mu_{2}-\eta^{1}\left(\mathrm{C}_{\alpha}\right): \eta^{1}\left(\mathrm{C}_{\alpha}\right)-\kappa^{1}(\mathrm{~N})-\mathrm{C}_{\alpha}=\mathrm{C}_{\beta}(\mathrm{H})\left(2-\mathrm{C}_{5} \mathrm{H}_{4} \mathrm{~N}\right)\right\}(\mu-\mathrm{CO}) \mathrm{PdI}\right]$. $\mathrm{B}$ случае сочетания $\mathrm{Cp}(\mathrm{CO})_{2} \mathrm{FeI}$ и метилпропиолата в таких же условиях (табл. 1 , реакции 3 и 4) протекания побочных реакций не наблюдалось, хотя предполагалось, что наличие метоксикарбонильной группы в исходном алкине, способной координировать атом металла по атому кислорода карбонильной группы, будет способствовать образованию аналогичного биядерного $\mu$-винилиденового FePd комплекса.

Стоит обратить внимание, что примеры синтеза этинилметоксикарбонильного комплекса железа 1 приводили и в других работах, однако его выходы нигде не превышали 50 \%. Например, $\mathrm{Cp}(\mathrm{CO})_{2} \mathrm{Fe}-\mathrm{C} \equiv \mathrm{C}-\mathrm{COOMe}(\mathbf{1})$ был получен по реакции $\left[\mathrm{Cp}(\mathrm{CO})_{2} \mathrm{Fe}_{[}\right]\left[\mathrm{BF}_{4}\right]$ и $\mathrm{H}-\mathrm{C} \equiv \mathrm{C}-\mathrm{COOMe}$ в присутствии избытка гидрокарбоната натрия с выходом 27 \% [17], по CuI-катализируемой реакции сочетания $\mathrm{Cp}(\mathrm{CO})_{2} \mathrm{FeI}$ и $\mathrm{H}-\mathrm{C} \equiv \mathrm{C}-\mathrm{COOMe}\left(0,6\right.$ моль \% $\mathrm{CuI}_{2} \mathrm{NEt}_{3}, 12$ ч) с выходом $45 \%$, а также с выходом $5 \%$ по реакции $\mathrm{Cp}(\mathrm{CO})_{2} \mathrm{FeBr}$ и $\mathrm{Li}-\mathrm{C} \equiv \mathrm{C}-\mathrm{COOMe} \mathrm{[18].}$

Таким образом, показана возможность успешного использования предложенных нами ра-

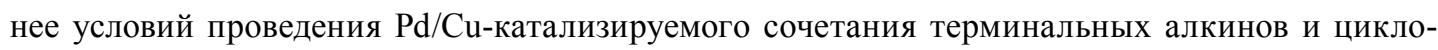
пентадиенилдикарбонилиодида железа для получения этинилметоксикарбонильного комплексажелеза 1. Предложенный нами метод позволяет получать комплекс $\mathbf{1}$ с количественными выходами (84-92 \%), которые, согласно литературным данным, ранее не были достигнуты. Установлено, что реакция $\mathrm{Pd} / \mathrm{Cu}-$-катализируемого сочетания $\mathrm{Cp}(\mathrm{CO})_{2} \mathrm{FeI}$ и метилпропиолата менее чувствительна к выбору основания и позволяет получать $\mathrm{Cp}(\mathrm{CO})_{2} \mathrm{Fe}-\mathrm{C} \equiv \mathrm{C}-\mathrm{COOMe}(\mathbf{1})$ как с использованием 1,8-диазобицикло[5.4.0]ундец-7-ена, так и с диизопропиламином (но с меньшим выходом).

\section{Благодарности / Acknowledgements}

Исследование выполнено за счет гранта Российского научного фонда (проект № 18-7300150). Работа выполнена с использованием оборудования Красноярского регионального центра коллективного пользования ФИЦ КНЦ СО РАН.

This research was funded by a grant from the Russian Science Foundation (project No. 1873-00150). Physical-chemical characteristics were obtained in the Krasnoyarsk Regional Centre of Research Equipment, Siberian Branch of the Russian Academy of Sciences.

\section{Список литературы / References}

1. Low P.J. Twists and turns: Studies of the complexes and properties of bimetallic complexes featuring phenylene ethynylene and related bridging ligands. Coord. Chem. Rev. 2013. Vol. 257(9-10), P. $1507-1532$.

2. Grelaud G. et al. Group 8 metal alkynyl complexes for nonlinear optics. J. Organomet. Chem. 2014. Vol. 751, P. 181-200.

3. Powell C.E., Humphrey M.G. Nonlinear optical properties of transition metal acetylides and their derivatives. Coord. Chem. Rev. 2004. Vol. 248 (7-8), P. 725-756. 
4. Yam V.W.-W., Au V.K.-M., Leung S.Y.-L. Light-Emitting Self-Assembled Materials Based on d 8 and d 10 Transition Metal Complexes. Chem. Rev. 2015. Vol. 115(15), P. 7589-7728.

5. Valyaev D.A., Semeikin O.V. Ustynyuk N.A. Redox induced reactions of transition metal vinylidene and related complexes. Coord. Chem. Rev. 2004. Vol. 248, P. 1679-1692.

6. Kaim V., Kaur-Ghumaan S. Manganese Complexes: Hydrogen Generation and Oxidation. Eur. J. Inorg. Chem. 2019. Vol. 2019(48), P. 5041-5051.

7. Long N.J., Williams C.K. Metal alkynyl $\sigma$ complexes: Synthesis and materials. Angew. Chemie - Int. Ed. 2003. Vol. 42(23), P. 2586-2617.

8. Bruce M.I., Ke M., Low P.J. Syntheses of metal diynyl complexes: novel routes to all-carbon ligands. Chem. Commun. 1996. Vol. 3(21), P. 2405-2406.

9. Hashmi A.S.K., Molinari L. Effective Transmetalation from Gold to Iron or Ruthenium. Organometallics. 2011. Vol. 30(13), P. 3457-3460.

10. Lo Sterzo C. The Wonder of Palladium Catalysis: From Carbon-Carbon to Metal-Carbon Bond Formation. An Opportunity of Getting Astonishment from Reality. Synlett. 1999. Vol. 1999(11), P. 1704-1722.

11. Nakaya R. et al. Synthesis of (1-alkynyl)dicarbonylcyclopentadienyliron complexes by palladium-catalyzed Sonogashira-type carbon-iron bond formation. Tetrahedron Lett. 2009. Vol. 50(37), P. 5274-5276.

12. Verpekin V.V. et al. Crystal structure of $\mu$-carbonyl-1:2 $\kappa^{2} \mathrm{C}: \mathrm{C}$-carbonyl- $1 \kappa \mathrm{C}-\left(1 \eta^{5}-\right.$ cyclopentadienyl)iodido-2 $\kappa \mathrm{I}$-[ $\mu$-2-(pyridin-2-yl)ethene-1,1-diyl- $\left.1 \kappa \mathrm{C}^{1}: 2 \kappa^{2} \mathrm{~N}, \mathrm{C} 1\right]$ ironpalladium(Fe-Pd) benzene monosolvate. Acta Crystallogr. Sect. E Crystallogr. Commun. 2017. Vol. 73, P. 68-71.

13. Verpekin V.V. et al. Catalyzed $\mathrm{M}-\mathrm{C}$ coupling reactions in the synthesis of $\sigma$-(pyridylethynyl) dicarbonylcyclopentadienyliron complexes. RSC Adv. 2020. Vol. 10(29), P. 17014-17025.

14. Anderson G.K. et al. Bis(Benzonitrile)Dichloro Complexes of Palladium and Platinum. Inorganic synthesis, Vol. 28 / ed. Angelici R.J. 1990. P. 60-63.

15. King R.B. et al. Cyclopentadienyl Metal Carbonyls and Some Derivatives. Inorganic Syntheses, Vol. 7 / ed. Jacob Kleinberg. John Wiley \& Sons, Ltd, 1963. P. 99-115.

16. Karak M., Barbosa L.C.A., Hargaden G.C. Recent mechanistic developments and next generation catalysts for the Sonogashira coupling reaction. RSC Adv. 2014. Vol. 4(96), P. 53442-53466.

17. Shaw M.J. et al. Synthesis and Electrochemistry of Iron-Pyrylium Complexes. Organometallics 2004. Vol. 23(11), P. 2778-2783.

18. Pilar Gamasa M. et al. Synthesis and characterization of novel $\sigma$-alkynyl cyclopentadienyl iron(II) complexes $\left[\mathrm{Fe}(\mathrm{C} \equiv \mathrm{CR}) \mathrm{L}_{2}\left(\eta-\mathrm{C}_{5} \mathrm{H}_{5}\right)\right]\left[\mathrm{L}=\mathrm{CO} ; \mathrm{L}_{2}=\right.$ bis(diphenylphosphino)methane (dppm)]. Crystal structure of $\left[\mathrm{Fe}\left(\mathrm{C} \equiv \mathrm{CC}_{6} \mathrm{H}_{5}\right)(\mathrm{dppm})\left(\eta-\mathrm{C}_{5} \mathrm{H}_{5}\right)\right]$. J. Organomet. Chem. 1991. Vol. 405(3). P. 333-345. 